\title{
Controlling polyelectrolyte adsorption onto carbon nanotubes by tuning ion-image interactions
}

\author{
Alpha A. Lee* \\ Cavendish Laboratory, University of Cambridge, Cambridge CBз OHE, UK \\ Sarah V. Kostinsk and Michael P. Brenner \\ School of Engineering and Applied Sciences, Harvard University, Cambridge, MA 02138, USA.
}

\begin{abstract}
Understanding and controlling polyelectrolyte adsorption onto carbon nanotubes is a fundamental challenge in nanotechology. Polyelectrolytes have been shown to stabilise nanotube suspensions through adsorbing onto the nanotube surface, and polyelectrolyte-coated nanotubes are emerging as building blocks for complex and addressable self-assembly. The conventional wisdom suggests that polyelectrolyte adsorption onto nanotubes is driven by specific chemical or van der Waals interactions. We develop a simple mean-field model and show that ion-image attraction is a significant effect for adsorption onto conducting nanotubes at low salt concentrations. Our theory suggests a simple strategy to selectively and reversibly functionalize carbon nanotubes based on their electronic structure which in turn modifies the ion-image attraction.
\end{abstract}

\section{INTRODUCTION}

Carbon nanotubes (CNTs) are one of the most important and ubiquitous building blocks in nano devices [1]3. Their plethora of applications range from transistors [4, 5] and electrical wires [6, 7] to probes for in vitro imaging of biological systems [8 10. Exploiting the interaction between polyelectrolytes and CNTs is a recurring theme in many of those applications: CNTs can be dispersed in solution via polyelectrolyte adsorption [11-13] and conducting CNTs can be separated from the semiconducting CNTs [14 16, although how the electronic structure of CNTs affects adsorption is hitherto less clear. Polyelectrolyte-nanotube complexes can themselves be used as chemical sensors [17, electro-optical materials [18, as well as building blocks for complex selfassembled structure [19] such as plasmonic metamaterials 20. Moreover, understanding the interaction of CNTs with biopolymers, which are often charged, is important when assessing the in vitro toxicity of CNTs 21.

Previous theoretical studies and simulations explain polyelectrolyte adsorption onto CNTs in terms of specific chemical interactions, such as $\pi-\pi$ stacking between aromatic monomers (e.g. nucleobases in the case of DNA) and the nanotube surface [22 27. This microscopic picture is deduced from classical molecular dynamics simulations of CNT-polyelectrolyte interactions which neglects the electronic structure of the CNT, perhaps because the development of efficient simulation techniques to account for metallic surfaces is still ongoing [28 31, and use Lennard-Jones potentials to model CNT-polyelectrolyte interactions. Those pioneering works show that specific chemical affinity is a sufficient condition for adsorption. Nonetheless, they raise the broader questions of whether adsorption is unique to polyelectrolytes with significant nonelectrostatic interactions and what role does the electronic structure of CNTs play in driving adsorption.
In this paper, we argue that there is another significant effect driving adsorption - the ion-image attraction. The ion-image attraction arises as external charges polarise electrons on the surface of a conductor. This induces an equal and opposite surface charge which lowers the selfenergy of ions near conducting boundaries, thus attracts ions closer to the surface of a conductor 32. Unlike specific chemical interactions, the ion-image attraction is a universal feature between ions and electrical conductors, and its strength and range depends on the electronic structure of the material 33. An emerging body of simulations using constant potential surfaces have already demonstrated the importance of including ion-image interactions to understand the physics of ions near interfaces, such as the arrangement of ions near metallic electrodes [34, 35], in carbon nanopores [36 39], as well as polyelectrolyte adsorption onto a planar surface with a low dielectric constant where the ion-image interaction is repulsive [40. Moreover, recent experiments directly revealed the effect of the electronic structure of the substrate on hydrodynamic flows in nanotubes [41, 42] and phase behaviour of electrolytes under nanoconfinement [43. However, the role of ion-image interaction in polyelectrolyte adsorption onto CNTs remains unexplored.

We will first introduce a mean-field theory for polyelectrolyte adsorption, and show that ion-image interactions can dominate over van der Waals interactions at low salt concentrations. We will then map the adsorption phase diagram, demonstrating how salt concentration could be tuned to allow selective adsorption of polyelectrolytes depending on the electronic structure of the CNT. The goal of our paper is to present a physical picture of how ionimage interactions could be a simple handle to control polyelectrolyte adsorption onto CNT, via estimates of the van der Waals and ion-image energies. As such, a simplified but analytically tractable model will be used. 


\section{THEORY}

We model the polyelectrolyte as a polymer with linear charge density $\rho$ with $N$ freely hinged links of Kuhn length $l$ each. The CNT exerts a potential $U(\mathbf{r})$ on each segment. In the continuum limit, the probability density $G\left(\mathbf{r}, \mathbf{r}_{0}, L\right)$ (Green's function) with one end point located at $\mathbf{r}$ and the other end point located at $\mathbf{r}_{0}$ is given by the equation [44 50 ]

$$
\left[\frac{\partial}{\partial N}-\frac{l^{2}}{6} \Delta_{\mathbf{r}}+\beta U(\mathbf{r})\right] G\left(\mathbf{r}, \mathbf{r}_{0}, L\right)=\delta\left(\mathbf{r}-\mathbf{r}_{0}\right) \delta(N) .
$$

where $\beta=k_{B} T$. The probability of finding the polymer approaches zero at infinity and at a hard wall, thus

$$
\lim _{|\mathbf{r}| \rightarrow \infty} G(\mathbf{r})=0, \text { and } G\left(\mathbf{r}_{s}\right)=0 .
$$

where $\mathbf{r}_{s}$ is the location of the CNT surface. $G\left(\mathbf{r}, \mathbf{r}_{0}, L\right)$ can be expressed in terms of a bilinear expansion

$$
G\left(\mathbf{r}, \mathbf{r}_{0}, L\right)=\sum_{n=0}^{\infty} f_{n}(\mathbf{r}) f_{n}^{*}\left(\mathbf{r}_{0}\right) e^{-\lambda_{n} N}
$$

where $f_{n}(\mathbf{r})$ and $\lambda_{n}$ are given by the eigenvalue problem

$$
\left[-\frac{l^{2}}{6} \Delta_{\mathbf{r}}+\beta U(\mathbf{r})\right] f_{n}(\mathbf{r})=\lambda_{n} f_{n}(\mathbf{r}) .
$$

In the limit of a long polymer chain, $N \gg 1$, the lowest eigenvalue dominates Equation (3) and $G\left(\mathbf{r}, \mathbf{r}_{0}, L\right) \approx$ $f_{0}(\mathbf{r}) f_{0}\left(\mathbf{r}_{0}\right) e^{-\lambda_{0} N}$. Therefore, the polymer is adsorbed to the surface if and only if a bound state exists, which corresponds to $\lambda_{0}<0$.

To compute the eigenvalues of Equation (4), we need to estimate the polyelectrolyte-CNT interaction potential $U(\mathbf{r})$ and the persistence length $l$. First we will consider the ion-image contribution $\left(U_{i i}(\mathbf{r})\right)$ to $U(\mathbf{r})$ and later we will estimate the strength of the van der Waals contribution. We ignore specific chemical interactions as our goal is to estimate how ion-image interactions, which is an universal feature of charges near conducting interfaces, could drive adsorption. $U_{i i}(\mathbf{r})$ can be estimated by considering a point charge $q$ of the polyelectrolyte located at distance $\mathbf{r}_{\mathbf{q}}$, where $r=0$ defines the axis of the CNT which we model as an infinitely long cylinder with radius $a$. Assuming an uniform dielectric constant $\varepsilon$, within the Debye-Hückel approximation the potential $\phi$ satisfies

$$
\nabla^{2} \phi-\kappa_{D}^{2} \phi=-\frac{q}{\varepsilon} \delta\left(\mathbf{r}-\mathbf{r}_{\mathbf{q}}\right),
$$

where $\kappa_{D}=\sqrt{8 \pi l_{B} c}$ is the inverse Debye length, with $l_{B}=e^{2} /\left(4 \pi \epsilon k_{B} T\right)$ the Bjerrum length and $c$ the salt concentration. The electronic structure of the CNT enters into the electrostatic boundary value problem via the metallicity (also known in the literature as the quantum capacitance [51]). The metallicity arises as one of the electrostatic boundary conditions: the electric field inside and outside the CNT is related to the induced charge $\rho_{\text {ind }}$ via Gauss' law

$$
\varepsilon[\mathbf{n} \cdot \nabla \phi]=-\frac{\rho_{\text {ind }}}{2 \pi a}=\frac{C_{q} \phi_{s}}{2 \pi a},
$$

where $[\cdot]$ denotes the jump across the CNT surface, $\mathbf{n}$ is the unit vector normal to the surface (defined to point in the direction outside of the pore). To arrive at the second equality, we assumed linear response, thus the induced charge is proportional to the potential at the surface, $\phi_{s}$, with the constant of proportionality $C_{q}$ being the metallicity [52]. The classical boundary condition for an ideal metal, $\phi_{s}=0$, is recovered for $C_{q}=\infty$. Solution of Equation (5) with boundary condition (6) and the conditions of global continuity and asymptotic decay of the potential [32] can be found via standard Fourier transform techniques.

The ion-image interaction arises because the presence of a metallic boundary lowers the self-energy of ions. The self-energy of ions is lower if they are closer to the metallic boundary, thus the ions experience a body force. The self-energy is related to the potential via

$$
E_{\text {self }}\left(\mathbf{r}_{q}\right)=q \lim _{\mathbf{r} \rightarrow \mathbf{r}_{q}}\left[\phi\left(\mathbf{r}, \mathbf{r}_{q} ; C_{q}\right)-\phi\left(\mathbf{r}, \mathbf{r}_{q} ; 0\right)\right]
$$

Substituting the solution of Equation (5) into (7), and noting that the polyelectrolyte is continuous and assumed to be homogeneously charged, we arrive at the ion-image interaction energy density

$$
\beta U_{i i}(R)=-\frac{l_{B}}{\pi a}\left(\frac{\rho l}{e}\right)^{2} \sum_{m=-\infty}^{\infty} \int_{-\infty}^{\infty} \mathrm{d} x\left[\frac{C I_{m}^{2}\left(\sqrt{x^{2}+\left(\kappa_{D} a\right)^{2}}\right) K_{m}^{2}\left(\sqrt{x^{2}+\left(\kappa_{D} a\right)^{2}} R\right)}{1+C I_{m}\left(\sqrt{x^{2}+\left(\kappa_{D} a\right)^{2}}\right) K_{m}\left(\sqrt{x^{2}+\left(\kappa_{D} a\right)^{2}}\right)}\right]
$$

where we have introduced dimensionless quantities $C=$ $C_{q} /(2 \pi \varepsilon), R=r / a$, and $K_{m}$ and $I_{m}$ are the $m^{\text {th }}$ modified Bessel function of the first and second kind.

To close the problem, we now turn to estimate the per- sistence length $l$, which accounts for the mechanical stiffness of the polymer chains as well as the intrapolymer electrostatic interactions. For a worm-like chain polyelectrolyte, the Odijk-Skolnick-Fixman theory gives the 
simple expression 53,54

$$
l=l_{0}+\frac{l_{B}}{4 A^{2} \kappa_{D}^{2}},
$$

where $l_{0}$ is the bare persistence length, and $A$ the distance between elementary charges along the chain $\left(l_{B}\right.$ in the case of Manning condensation). Equation (9) contains the salient physics that like-charge repulsion between charge groups on the polyelectrolyte increases stiffness, thus the persistence length is a decreasing function of the Debye length.

The system of equations, Equations (4), (8) and (9), is the salient result of this paper. Those equations relate the sign of the ground state eigenvalue, $\lambda_{0}$, to the dimensionless parameters $\kappa a, C_{q} / 2 \pi \varepsilon$, and $l_{B} \rho l / \pi a e$. We consider typical parameters of $C_{q} \approx 2 \times 10^{-10} \mathrm{~F} / \mathrm{m}$ [55, 56], $a \approx 0.4 \mathrm{~nm}, c \approx 1 \mathrm{mM}$, and $\epsilon=80, l_{0}=50 \mathrm{~nm}$ [57. (parameters for double stranded DNA are used as a typical estimate for polyelectrolytes), with effective linear charge density $\rho=1 / l_{B}$ to account for Manning counterion condensation.

\section{RESULTS}

Figure 1 $1 \mathrm{a}-\mathrm{b}$ shows that the strength of the ion-image interaction energy, Equation (8), is controlled by the salt concentration and metallicity. The ion-image interaction is stronger for metallic CNTs with larger metallicity thus higher concentration of free electrons on the CNT surface to respond to the external charge. Increasing the ion concentration decreases the ion-image interaction as counterions screens the electric field from the polyelectrolyte ions. For comparison we also plot an estimate of the van der Waals interaction between a $(6,6) \mathrm{CNT}$ and $\mathrm{GC}(10)$ dsDNA in water using the Gecko Hamaker software tool [58], showing that the ion-image interaction is significantly larger in magnitude, even at distances much larger than the nanotube size. At low salt concentrations (long screening lengths) the ion-image interactions become a dominant source of polyelectrolyte-CNT interactions.

The dependence of the ion-image interaction on salt concentration and metallicity can be used to control polyelectrolyte adsorption. Figure 2 shows the phase diagram for the adsorption-desorption transition as a function of the metallicity and the inverse Debye length for parameters corresponding to dsDNA. Importantly, tuning the salt concentration allows a metallicity-selective adsorption of polyelectrolytes onto CNTs. At high salt concentration, the ion-image interaction is screened by counterions, thus polymers can only adsorb on CNTs with a large metallicity as the ion-image interaction energy for CNTs with lower metallicity is insufficient to hold the polyelectrolyte at the nanotube surface.

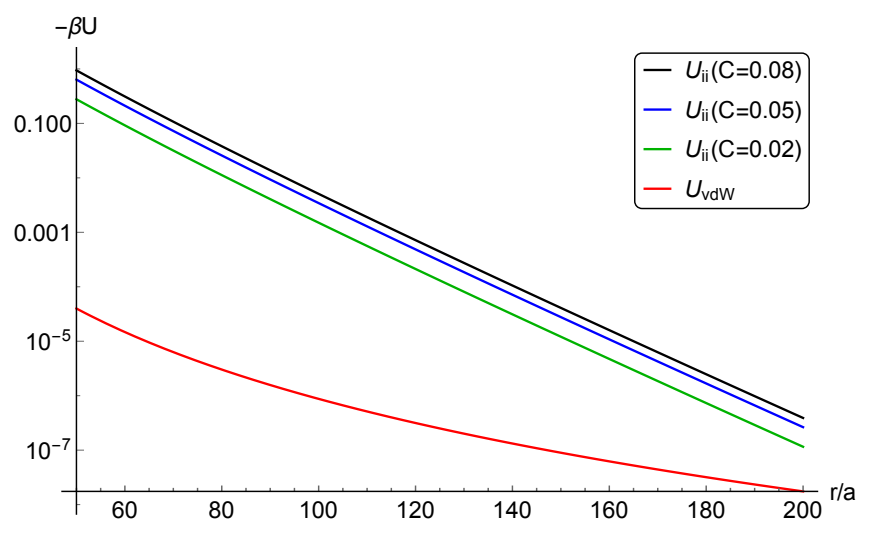

(a)

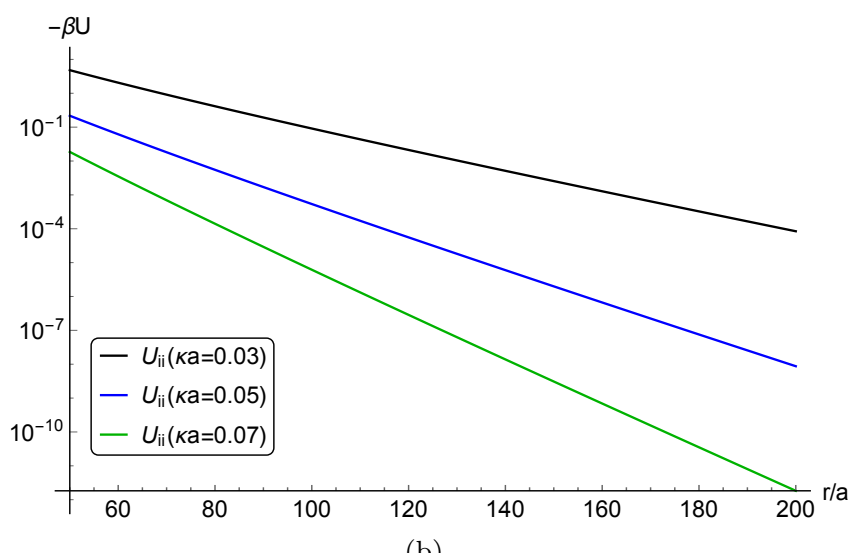

(b)

FIG. 1. The strength of the ion-image interaction energy, Equation (8), is controlled by the (a) metallicity and (b) salt concentration. All variables except the independent variable is set to the typical values estimated in the main text. The ion-image interaction energy is significantly stronger in magnitude compared to the van der Waals energy for distances $r / a \lesssim 240$. Lower salt concentrations (corresponding to lower $\kappa a)$ lessen the electrostatic screening factor and thus are preferred for stronger ion-image interactions.

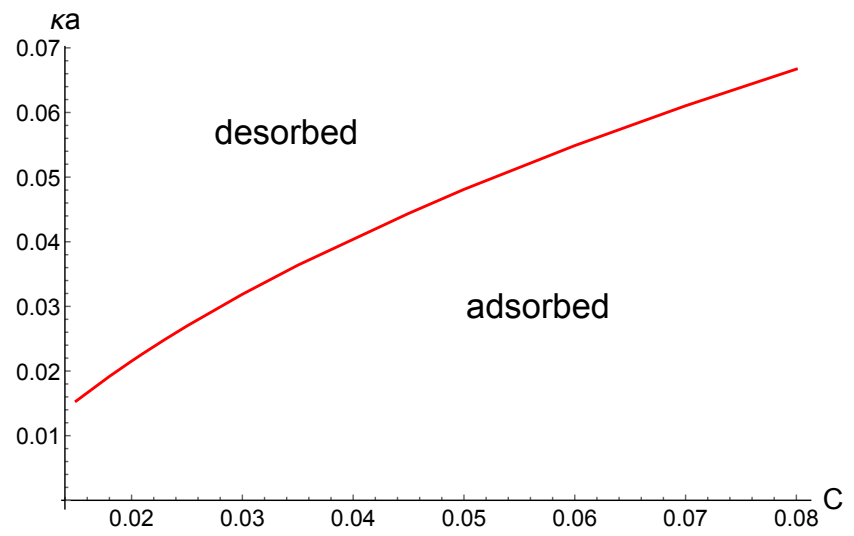

FIG. 2. Adsorption-desorption phase diagram: Tuning the salt concentration (i.e. $\kappa a$ ) allows metallicity-selective adsorption of polyelectrolytes onto CNTs. The curve is plotted with the typical parameters estimated in the main text. 


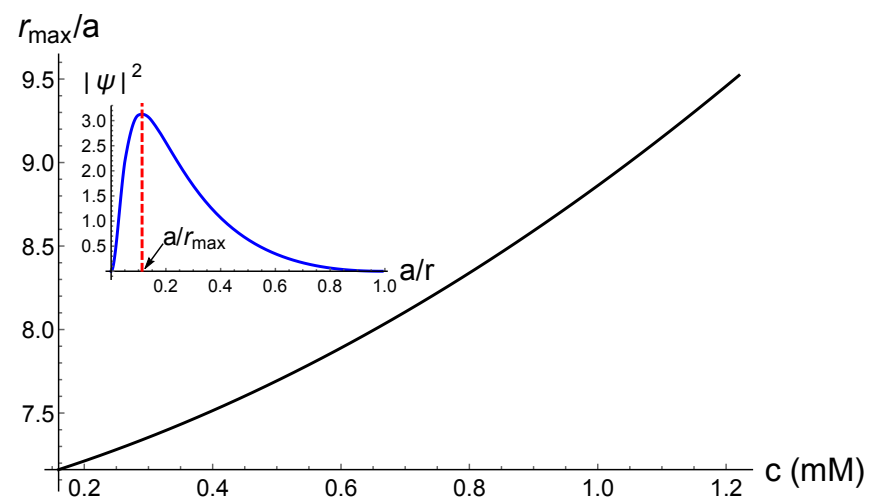

FIG. 3. The thickness of the adsorbed layer, denoted by $r_{\max }$, increases with salt concentration. Plotted above for a $(6,6)$ CNT with radius $a=0.4068 \mathrm{~nm}$ and $C=C_{q} / 2 \pi \varepsilon=0.05$. Inset: The $c=1 \mathrm{mM}$ probability distribution of monomers near the CNT surface, which has a maximum at $r_{\max }$.

The interplay between Debye screening and ion-image interaction can also be exploited to control the thickness of the adsorbed polyelectrolyte layer. Figure 3 shows the thickness of the adsorbed polyelectrolyte layer increases for increasing salt concentration. We take the thickness of the adsorbed layer to be the location of the peak in the probability distribution of monomers near the CNT surface (c.f. the inset of Figure (3p).

In deriving our model, Equations (4), (8) and (9), we have made several assumptions which we will now comment on. First, we considered the weak adsorption regime, where the entropic free energy of polyelectrolyte is comparable to the ion-image interaction with the CNT and thus interactions with the CNT only weakly perturbs the conformation of the chain [59]. This is expected to hold near the vicinity of the adsorption-desorption transition, which is the subject of this paper. Second, electrostatic interactions is treated using Debye-Hückel theory, which is a mean-field approximation only valid in the asymptotic limit of dilute electrolyte, $l_{B}^{3} c \ll 1$. The adsorption-desorption transition occurs at $O(\mathrm{mM})$, thus $l_{B}^{3} c=O\left(10^{-4}\right)$ and the mean field approximation is reasonable. Third, we assumed that the polyelectrolyte profile is built up near the adsorbing surface without disturbing the electrostatic potential and ionic distribution near the interface. This approximation is made to render calculations analytically tractable. For constant charge surfaces, a more general self-consistent field theory has been presented in refs. 60, 61, although a self-consistent computation of just ion-ion interactions near a metallic surface is considerably more complex 62 65, let alone for a polyelectrolyte system. Fourth, we modelled the CNT as an infinite long cylinder. This assumption is justified when the persistence length of the polyelectrolyte is much greater than the persistence length of the CNT (typically $O(100 \mu \mathrm{m})$ 66]), which holds for the example of double stranded DNA considered here. We have also neglected the rod-like nature of the polyelectrolyte segments in computing the ion-image interaction energy. Finally, the electronic structure of the CNT is modelled using a linearised metallicity (quantum capacitance). For sufficiently large surface potential, the metallicity becomes potential-dependent. In the non-linear regime, the superposition property of linear partial differential equations which we relied on to relate the ion-image interaction energy of a point charge to the ion-image interaction energy of a polyelectrolyte is invalid.

\section{DISCUSSION AND CONCLUSIONS}

In summary, we showed how ion-image interaction could drive polyelectrolyte adsorption onto carbon nanotube independent of any chemical affinities between the monomers and the nanotube. The ion-image interaction is tunable by varying the salt concentration and is strongly dependent on the electronic structure of the nanotube. As such, varying the salt concentration is a simple strategy to selectively and reversibly functionalise of carbon nanotubes based on their electronic structure, and the phase diagram mapped by our theory suggests relevant regions of the parameter space. Experiments and simulations which systematically the effect of metallicity on polyelectrolyte adsorption is scarce; we hope that our theory provides a framework to design experiments and motivate further investigation. In particular, ref [43] reported a set of experimentally viable surfaces with varying metallicity, and extending this pioneering work to investigate polyelectrolyte adsorption would be an exciting direction.

AAL was supported by the George F. Carrier Fellowship at Harvard University and acknowledges the Winton Programme for the Physics of Sustainability at the University of Cambridge for funding. SVK was supported by the Department of Defense (DoD) through the National Defense Science \& Engineering Graduate Fellowship (NDSEG) Program, and by National Science Foundation grant DMS-1411694. MPB is an Investigator of the Simons Foundation.

* aal44@cam.ac.uk

$\dagger$ AAL and SVK contributed equally to this work.

‡arah.kostinski@mail.huji.ac.il AAL and SVK contributed equally to this work.

[1] Schnorr, J. M.; Swager, T. M. Emerging applications of carbon nanotubes. Chemistry of Materials 2010, 23, 646-657.

[2] Dresselhaus, M. S.; Dresselhaus, G.; Avoruis, P. Carbon nanotubes: synthesis, structure, properties, and applications; Springer Science \& Business Media, 2003; Vol. 80.

[3] Zhang, Q.; Huang, J.-Q.; Qian, W.-Z.; Zhang, Y.-Y.; Wei, F. The road for nanomaterials industry: A review of 
carbon nanotube production, post-treatment, and bulk applications for composites and energy storage. Small 2013, 9, 1237-1265.

[4] Tans, S. J.; Verschueren, A. R.; Dekker, C. Roomtemperature transistor based on a single carbon nanotube. Nature 1998, 393, 49-52.

[5] Martel, R.; Schmidt, T.; Shea, H.; Hertel, T.; Avouris, P. Single-and multi-wall carbon nanotube field-effect transistors. Applied Physics Letters 1998, 73, 2447-2449.

[6] others, , et al. Carbon nanotubes as molecular quantum wires. Physics today 1999, 52, 22-30.

[7] Kurzepa, L.; Lekawa-Raus, A.; Patmore, J.; Koziol, K. Replacing copper wires with carbon nanotube wires in electrical transformers. Advanced Functional Materials 2014, 24, 619-624.

[8] Fakhri, N.; Wessel, A. D.; Willms, C.; Pasquali, M.; Klopfenstein, D. R.; MacKintosh, F. C.; Schmidt, C. F. High-resolution mapping of intracellular fluctuations using carbon nanotubes. Science 2014, 344, 1031-1035.

[9] Hong, G.; Diao, S.; Antaris, A. L.; Dai, H. Carbon nanomaterials for biological imaging and nanomedicinal therapy. Chemical reviews 2015, 115, 10816-10906.

[10] Battle, C.; Broedersz, C. P.; Fakhri, N.; Geyer, V. F.; Howard, J.; Schmidt, C. F.; MacKintosh, F. C. Broken detailed balance at mesoscopic scales in active biological systems. Science 2016, 352, 604-607.

[11] Liu, Y.; Gao, L.; Zheng, S.; Wang, Y.; Sun, J.; Kajiura, H.; Li, Y.; Noda, K. Debundling of single-walled carbon nanotubes by using natural polyelectrolytes. Nanotechnology 2007, 18, 365702.

[12] Deria, P.; Sinks, L. E.; Park, T.-H.; Tomezsko, D. M.; Brukman, M. J.; Bonnell, D. A.; Therien, M. J. Phase transfer catalysts drive diverse organic solvent solubility of single-walled carbon nanotubes helically wrapped by ionic, semiconducting polymers. Nano letters 2010, 10, 4192-4199.

[13] Samanta, S. K.; Fritsch, M.; Scherf, U.; Gomulya, W.; Bisri, S. Z.; Loi, M. A. Conjugated polymer-assisted dispersion of single-wall carbon nanotubes: the power of polymer wrapping. Accounts of chemical research 2014, 47, 2446-2456.

[14] others,, et al. Structure-based carbon nanotube sorting by sequence-dependent DNA assembly. Science 2003, 302, 1545-1548.

[15] Zheng, M.; Jagota, A.; Semke, E. D.; Diner, B. A.; Mclean, R. S.; Lustig, S. R.; Richardson, R. E.; Tassi, N. G. DNA-assisted dispersion and separation of carbon nanotubes. Nature materials 2003, 2, 338-342.

[16] Strano, M. S.; Zheng, M.; Jagota, A.; Onoa, G. B.; Heller, D. A.; Barone, P. W.; Usrey, M. L. Understanding the nature of the DNA-assisted separation of singlewalled carbon nanotubes using fluorescence and Raman spectroscopy. Nano Letters 2004, 4, 543-550.

[17] Staii, C.; Johnson, A. T.; Chen, M.; Gelperin, A. DNAdecorated carbon nanotubes for chemical sensing. Nano Letters 2005, 5, 1774-1778.

[18] Deria, P.; Von Bargen, C. D.; Olivier, J.-H.; Kumbhar, A. S.; Saven, J. G.; Therien, M. J. Single-handed helical wrapping of single-walled carbon nanotubes by chiral, ionic, semiconducting polymers. Journal of the American Chemical Society 2013, 135, 16220-16234.

[19] Maune, H. T.; Han, S.-p.; Barish, R. D.; Bockrath, M.; Goddard III, W. A.; Rothemund, P. W.; Winfree, E. Selfassembly of carbon nanotubes into two-dimensional ge- ometries using DNA origami templates. Nature nanotechnology 2010, 5, 61-66.

[20] Tan, S. J.; Campolongo, M. J.; Luo, D.; Cheng, W. Building plasmonic nanostructures with DNA. Nature nanotechnology 2011, 6, 268-276.

[21] Madani, S. Y.; Mandel, A.; Seifalian, A. M. A concise review of carbon nanotube's toxicology. Nano reviews 2013, 4, 21521.

[22] Gao, H.; Kong, Y. Simulation of DNA-nanotube interactions. Annu. Rev. Mater. Res. 2004, 34, 123-150.

[23] Zhao, X.; Johnson, J. K. Simulation of adsorption of DNA on carbon nanotubes. Journal of the American Chemical Society 2007, 129, 10438-10445.

[24] Johnson, R. R.; Johnson, A. C.; Klein, M. L. Probing the structure of DNA- carbon nanotube hybrids with molecular dynamics. Nano Letters 2008, 8, 69-75.

[25] Zhao, X. Self-assembly of DNA segments on graphene and carbon nanotube arrays in aqueous solution: a molecular simulation study. The Journal of Physical Chemistry $C$ 2011, 115, 6181-6189.

[26] Roxbury, D.; Mittal, J.; Jagota, A. Molecular-basis of single-walled carbon nanotube recognition by singlestranded DNA. Nano letters 2012, 12, 1464-1469.

[27] Iliafar, S.; Mittal, J.; Vezenov, D.; Jagota, A. Interaction of single-stranded DNA with curved carbon nanotube is much stronger than with flat graphite. Journal of the American Chemical Society 2014, 136, 12947-12957.

[28] Siepmann, J. I.; Sprik, M. Influence of surface topology and electrostatic potential on water/electrode systems. The Journal of chemical physics 1995, 102, 511-524.

[29] Reed, S. K.; Lanning, O. J.; Madden, P. A. Electrochemical interface between an ionic liquid and a model metallic electrode. The Journal of chemical physics 2007, 126, 084704.

[30] Raghunathan, A.; Aluru, N. Self-consistent molecular dynamics formulation for electric-field-mediated electrolyte transport through nanochannels. Physical Review E 2007, 76, 011202.

[31] Vatamanu, J.; Bedrov, D.; Borodin, O. On the application of constant electrode potential simulation techniques in atomistic modelling of electric double layers. Molecular Simulation 2017, 43, 838-849.

[32] Jackson, J. D. Classical electrodynamics; John Wiley \& Sons, 2007.

[33] Pastewka, L.; Järvi, T. T.; Mayrhofer, L.; Moseler, M. Charge-transfer model for carbonaceous electrodes in polar environments. Physical Review B 2011, 83, 165418.

[34] Tazi, S.; Salanne, M.; Simon, C.; Turq, P.; Pounds, M.; Madden, P. A. Potential-induced ordering transition of the adsorbed layer at the ionic liquid/electrified metal interface. The Journal of Physical Chemistry B 2010, $114,8453-8459$.

[35] Limmer, D. T.; Merlet, C.; Salanne, M.; Chandler, D.; Madden, P. A.; Van Roij, R.; Rotenberg, B. Charge fluctuations in nanoscale capacitors. Physical review letters 2013, 111, 106102.

[36] Wu, P.; Huang, J.; Meunier, V.; Sumpter, B. G.; Qiao, R. Complex capacitance scaling in ionic liquidsfilled nanopores. Acs Nano 2011, 5, 9044-9051.

[37] Merlet, C.; Rotenberg, B.; Madden, P. A.; Taberna, P.L.; Simon, P.; Gogotsi, Y.; Salanne, M. On the molecular origin of supercapacitance in nanoporous carbon electrodes. Nature materials 2012, 11, 306-310. 
[38] Merlet, C.; Péan, C.; Rotenberg, B.; Madden, P. A.; Simon, P.; Salanne, M. Simulating supercapacitors: can we model electrodes as constant charge surfaces? The journal of physical chemistry letters 2012, 4, 264-268.

[39] Wu, P.; Huang, J.; Meunier, V.; Sumpter, B. G.; Qiao, R. Voltage dependent charge storage modes and capacity in subnanometer pores. The journal of physical chemistry letters 2012, 3, 1732-1737.

[40] Netz, R. R.; Joanny, J.-F. Adsorption of semiflexible polyelectrolytes on charged planar surfaces: charge compensation, charge reversal, and multilayer formation. Macromolecules 1999, 32, 9013-9025.

[41] Secchi, E.; Marbach, S.; Niguès, A.; Stein, D.; Siria, A.; Bocquet, L. Massive radius-dependent flow slippage in carbon nanotubes. Nature 2016, 537, 210-213.

[42] Majumder, M.; Siria, A.; Bocquet, L. Flows in onedimensional and two-dimensional carbon nanochannels: Fast and curious. MRS Bulletin 2017, 42, 278-282.

[43] Comtet, J.; Niguès, A.; Kaiser, V.; Coasne, B.; Bocquet, L.; Siria, A. Nanoscale capillary freezing of ionic liquids confined between metallic interfaces and the role of electronic screening. Nature Materials 2017, 16, 634639.

[44] Edwards, S. F. The statistical mechanics of polymers with excluded volume. Proceedings of the Physical Society 1965, 85, 613.

[45] Lifshitz, I. Some problems of the statistical theory of biopolymers. Sov. Phys. JETP 1969, 28.

[46] Wiegel, F. Adsorption of a macromolecule to a charged surface. Journal of Physics A: Mathematical and General 1977, 10, 299.

[47] Doi, M.; Edwards, S. F. E. The theory of polymer dynamics; oxford university press, 1988; Vol. 73.

[48] Von Goeler, F.; Muthukumar, M. Adsorption of polyelectrolytes onto curved surfaces. The Journal of chemical physics 1994, 100, 7796-7803.

[49] Winkler, R. G.; Cherstvy, A. G. Critical adsorption of polyelectrolytes onto charged spherical colloids. Physical review letters 2006, 96, 066103.

[50] Cherstvy, A.; Winkler, R. Polyelectrolyte adsorption onto oppositely charged interfaces: unified approach for plane, cylinder, and sphere. Physical Chemistry Chemical Physics 2011, 13, 11686-11693.

[51] Luryi, S. Quantum capacitance devices. Applied Physics Letters 1988, 52, 501-503.

[52] Lee, A. A.; Vella, D.; Goriely, A. Quantum capacitance modifies interionic interactions in semiconducting nanopores. EPL (Europhysics Letters) 2016, 113, 38005.
[53] Odijk, T. Polyelectrolytes near the rod limit. Journal of Polymer Science Part B: Polymer Physics 1977, 15, 477-483.

[54] Skolnick, J.; Fixman, M. Electrostatic persistence length of a wormlike polyelectrolyte. Macromolecules 1977, 10, 944-948.

[55] Rotkin, S. V.; Subramoney, S. Applied physics of carbon nanotubes: fundamentals of theory, optics and transport devices; Springer Science \& Business Media, 2006.

[56] Pak, A. J.; Paek, E.; Hwang, G. S. Relative contributions of quantum and double layer capacitance to the supercapacitor performance of carbon nanotubes in an ionic liquid. Physical Chemistry Chemical Physics 2013, 15, 19741-19747.

[57] Baumann, C. G.; Smith, S. B.; Bloomfield, V. A.; Bustamante, C. Ionic effects on the elasticity of single DNA molecules. Proceedings of the National Academy of Sciences 1997, 94, 6185-6190.

[58] Rajter, R. F.; Podgornik, R.; Parsegian, V. A.; French, R. H.; Ching, W. van der Waals-London dispersion interactions for optically anisotropic cylinders: Metallic and semiconducting single-wall carbon nanotubes. Physical Review B 2007, 76, 045417.

[59] Cherstvy, A.; Winkler, R. Strong and weak adsorptions of polyelectrolyte chains onto oppositely charged spheres. The Journal of chemical physics 2006, 125, 064904.

[60] Podgornik, R. Self-consistent-field theory for confined polyelectrolyte chains. The Journal of Physical Chemistry 1992, 96, 884-896.

[61] Borukhov, I.; Andelman, D.; Orland, H. Effect of polyelectrolyte adsorption on intercolloidal forces. The Journal of Physical Chemistry B 1999, 103, 5042-5057.

[62] Hatlo, M. M.; Lue, L. The role of image charges in the interactions between colloidal particles. Soft Matter 2008, 4, 1582-1596.

[63] Hatlo, M. M.; Curtis, R. A.; Lue, L. Electrostatic depletion forces between planar surfaces. The Journal of chemical physics 2008, 128, 164717.

[64] Wang, R.; Wang, Z.-G. Effects of image charges on double layer structure and forces. The Journal of chemical physics 2013, 139, 124702.

[65] Wang, R.; Wang, Z.-G. Inhomogeneous screening near the dielectric interface. The Journal of chemical physics 2016, 144, 134902.

[66] Fakhri, N.; Tsyboulski, D. A.; Cognet, L.; Weisman, R. B.; Pasquali, M. Diameter-dependent bending dynamics of single-walled carbon nanotubes in liquids. Proceedings of the National Academy of Sciences 2009, 106, 14219-14223. 